\title{
Protée
}

\section{Présences réelles dans les mondes virtuels}

\section{Valérie Morignat}

Volume 34, numéro 2-3, automne-hiver 2006

Actualités du récit. Pratiques, théories, modèles

URI : https://id.erudit.org/iderudit/014264ar

DOI : https://doi.org/10.7202/014264ar

Aller au sommaire du numéro

Éditeur(s)

Département des arts et lettres - Université du Québec à Chicoutimi

ISSN

0300-3523 (imprimé)

1708-2307 (numérique)

Découvrir la revue

Citer cet article

Morignat, V. (2006). Présences réelles dans les mondes virtuels. Protée, 34(2-3), 41-52. https://doi.org/10.7202/014264ar

\section{Résumé de l'article}

Les arts numériques interactifs élaborent des stratégies immersives qui impliquent le spectateur dans un parcours historique et incarné à l'intérieur de mondes virtuels peuplés d'agents intelligents. En proposant les concepts d'interactantialité et de mésonarrativité dans l'examen du Cinéma numérique et du Jeu vidéo, cet article aborde les spécificités d’une narrativité numérique écologiquement située par laquelle le sujet réel partage les caractéristiques ontologiques du personnage de fiction. 


\title{
PRÉSEN CES RÉELLES D AN S LES M O N DES VIRTU ELS
}

\author{
VALÉRIE MORIGNAT
}

Pour moi, le monde futur ne sera pas un lieu mais un événement. Non
pas la construction d'un écrivain, des mots formant un roman ou une
nouvelle que les gens auront sous les yeux et qu'ils liront de l'extérieur
- mais plutôt une construction dans laquelle il n'y aurait ni auteurs ni
lecteurs, mais un grand nombre de personnages en quête d'une histoire.

(Philip K. Dick, 1988: 42)

Dans la conclusion d'Univers de la fiction, Thomas Pavel relevait la tendance remarquable consistant à réduire «la distance qui sépare le spectateur du monde fictionnel» (1988: 184). Les arts numériques interactifs amplifient ce fait en élaborant des stratégies immersives qui engagent le spectateur dans un parcours historique et incarné à l'intérieur de mondes virtuels.

La mise en intrigue devient alors, dans les environnements numériques, ce "phénomène-enveloppe» qu'évoquait Merleau-Ponty au sujet de l'organisme (1994: 275). Elle est désormais l'événement d'une communication intersensorielle opérée «entre les éléments» (ibid.) ${ }^{1}$, à l'intersection des actions du sujet et de la générativité des programmes. Semblable à l'organisme merleau-pontien, la narration interactée «ne dépasse la causalité que par le détour d'une réinterprétation d'une nouvelle dimensionnalité, par intégration et différenciations qualitatives" (ibid.: 276). Ainsi, c'est à une véritable ontogenèse du récit que nous conduira l'analyse des systèmes artificiels qui le mobilisent.

Dans le Cinéma interactif comme dans le Jeu vidéo, nous approcherons les modalités des processus narratifs interactés qui président à l'émergence d'environnements virtuels complexes. Lorsque l'intelligence artificielle (IA) - dans les systèmes multi-agents et les systèmes intentionnels - accompagne l'élaboration narrative, on verra que la trame évolutionnaire du vivant supporte des intrigues «émergentes» et «enchevêtrées", singulières «histoires non (encore) racontées» dont Ricœur suggérait l'existence dans Temps et Récit (1983: 142). Dans ce contexte, se profile une narration écologiquement située. 


\section{HISTOIRES POTENTIELLES}

Dans Temps et Récit, Paul Ricœur désigne par l'expression de "mise en intrigue» les opérations de configuration et les agencements qui actualisent une histoire. Décrivant ces procès instaurateurs, il leur attribue une dimension existentielle à travers de singulières interrogations:

[...] pourrions-nous parler d'une vie humaine comme d'une histoire à l'état naissant? [...] Sans quitter l'expérience quotidienne, ne sommes-nous pas inclinés à voir dans tel enchaînement d'épisodes de notre vie des histoires "non (encore) racontées", des histoires qui demandent à être racontées? (Ibid.: 141)

La mise en intrigue participerait donc de l'expérience vécue; elle serait à la fois ce qui en résulte et ce qui la façonne. Ricœur accorde en effet

[...] à l'expérience en tant que telle une narrativité inchoative qui ne procède pas de la projection, comme on dit, de la littérature sur la vie, mais qui constitue une authentique demande de récit. (Ibid., je souligne)

Ce postulat l'autorise alors à désigner une «structure pré-narrative de l'expérience» inscrite au cour même des actions incarnées, à l'échelle de la temporalité de la vie.

En situant la genèse de la narrativité dans le lieu de l'expérience, Ricœur suggère les rapports d'influence réciproque que l'expérience vécue entretiendrait avec son histoire virtuelle. Toute expérience vécue, nous ditil, serait ainsi une "histoire non encore racontée " (ibid.), une "histoire potentielle» (ibid.: 142). Les «histoires non encore dites de nos vies» (ibid.: 144) «imbrication vivante de toutes les histoires vécues les unes dans les autres» (ibid.: 143) - constitueraient alors l'arrière-pays génésique d'un récit perpétuellement en train de se faire, et faisant retour dans la vie même (ibid.: 141-144).

Aussi, Ricœur en vient à conclure que [...] la conséquence principale de cette analyse existentielle de l'homme comme "être enchevêtré dans des histoires» est que raconter est un processus secondaire [...]. Raconter, suivre, comprendre des histoires n'est que la "continuation" de ces histoires non dites. (Ibid.: 143, je souligne)
Ce passage de Temps et Récit est d'importance pour l'étude des modalités narratives dans les environnements numériques, car il vise une narrativité ontologique, solidaire de la vie d'où elle «émerge» et qu'elle reconfigure comme histoire potentielle. Plus encore, l'expérience vécue, d'une part, se double de sa mise en récit éventuelle, d'autre part, est révélée par toutes les histoires en suspens, cristallisées en elle, et dans lesquelles elle se déporte pour mieux se montrer.

Ce double enjeu trouve un écho puissant dans l'interactivité numérique où les productions sémiotiques et sémantiques, qui induisent de nouvelles formes narratives, reposent sur l'expérience vivante d'un environnement codéterminé par ses interacteurs. Dans ce contexte, l'expérience incarnée est en effet l'horizon d'émergence des événements, de leurs agencements, de leur signifiance et de leur persistance dans la forme des mondes virtuels.

Doté d'une actantialité ontologique, l'interacteur des environnements numériques fait plus qu'apporter son concours à une praxis artistique; il entraîne tout le champ narratif potentiel qu'il découpe sur le fond de son expérience. Examinant la relation du récit et de sa réception par le lecteur, Ricœur a d'ailleurs révélé cette nécessaire rencontre d'horizons:

[...] si la mise en intrigue peut être décrite comme un acte $d u$ jugement et de l'imagination productrice, c'est dans la mesure où cet acte est l'œuvre conjointe du texte et de son lecteur, comme Aristote disait que la sensation est l'œurre commune du senti et $d u$ sentant. (Ibid.: 145 , je souligne)

Dans notre cadre d'analyse, il s'agit de déplacer cette "fusion d'horizons» entre l'univers du texte et celui du lecteur dans la relation du monde virtuel émergent et de ses interactants.

\section{ACTION INTERFACÉE}

De ce qui est réel, on ne peut donner aucune explication claire si ce n'est à l'aide de quelque chose de fictif.

(J. Bentham cité par Laval, 1994: 5)

La structure pré-narrative de l'expérience, histoires potentielles incorporées par le sujet, constituerait 
l'appareil cognitif essentiel pour son implication ontologique dans un monde virtuel dont il devient le méta-concepteur ${ }^{2}$. On constatera en effet que la compétence fictionnelle du sujet est sollicitée à des fins d'incorporation du complexe interactif; plus on immerge le sujet comme actant au sein d'une histoire qu'il codétermine, plus il intègre le système interactif et s'investit dans l'émergence d'un monde virtuel complexe.

Avant d'aborder deux œuvres numériques - Si Poteris Narrare, Licet de Jean-Michel Bruyère (2002) et In Time de Cyrille C. de Laleu (2005) - qui confient au sujet l'essentiel de la mise en intrigue et sollicitent sa compétence fictionnelle, précisons l'architecture générale de l'interactivité et de ses interfaces.

Dans les environnements numériques interactifs immersifs (Réalité Virtuelle) ou interfacés (Réalité Augmentée) -, l'ordre de l'action et des agencements d'événements est celui de la symbiose. Chaque action du sujet engage une rétroaction numérique qui fait émerger, en même temps que de nouveaux comportements, un milieu codéterminé par les organismes en présence ${ }^{3}$. Actants réels et virtuels s'organisent mutuellement dans la constitution d'un écosystème artificiel à l'intérieur duquel chacun permet l'émergence de l'autre par le biais d'interfaces sensibles. Sous la forme de capteurs (de mouvement, de pression, de voix, etc.) et d'effecteurs (projections vidéo, émissions sonores, simulation kinesthésique, etc.), ces filtres de communication autorisent ce que la cybernétique a appelé des «boucles d'action et de rétroaction" entre les programmes informatiques et les organismes biologiques.

Dans ces environnements interfacés, «l'espace ne peut plus être conçu que comme celui de l'échange et où les pseudo-centres ne sont plus que des échangeurs» (Serres, 1972: 145). Constituant une intersensorialité cyberorganique, les interfaces rassemblent les agents biologiques et artificiels en une corporéité virtuelle distribuée qui inscrit l'action au sein d'un continuum informationnel.

Ce continuum est le siège de mouvements et d'échanges:

méthodes, modèles, résultats circulent partout en son sein, exportés ou importés de tous lieux en tous lieux, de manière incessante. (Ibid.: 10)

Cette zone intermédiaire, constituée dans le dialogue interfacé, déploie alors des collaborations inédites. Distribuée dans un réseau d'informations numériques, toute action engendre une écriture de données susceptibles d'être ultérieurement reconfigurées, ou actualisées par d'autres actants humains ou des agents virtuels autonomes.

L'action interceptée par les interfaces (et, avec elle, les émissions sensorielles et symboliques humaines) devient ainsi la materia prima du programme. Aussi divers et multiples que soient les signaux émis, le transcodage numérique leur confère d'égales propriétés génératives.

On remarquera avec Jean-Marie Schaeffer que

[...] les supports numériques accèdent à une ubiquité sémiotique complète, puisqu'ils peuvent encoder indifféremment des signes linguistiques, iconiques ou phoniques et les combiner entre eux.

(1999: 110)

Ainsi, la nageuse virtuelle de l'installation Ex-îles (2004) des designers Electronic Shadow 4 , les organismes de vie artificielle de l'aquarium interactif des artistes Sommerer et Mignonneau (A-Volve, 1994-1997) ou leurs biotopes d'Intro Act (1996) trouvent leur source génétique et leur évolutivité dans la multiplicité des actions et des émissions sensorielles humaines captées par les interfaces.

Modifiable, actualisable à l'infini, l'action interfacée accède à l'ubiquité sémiotique en même temps qu'à la puissance matricielle. Soustraite à sa propre temporalité, elle s'inscrit alors dans une dynamique de générativité sémiotique et informationnelle récursive, partagée entre toutes les entités du système. Dans le cadre du Cinéma interactif, c'est ce que Jeffrey Shaw a désigné comme l'émergence d'une multi-temporalité dans laquelle les actants font l'expérience des conséquences de leurs propres actions, de toutes celles qui les ont précédées, mais aussi de leurs transmutations, réécritures, réincarnations. 
Nous verrons que cette expérience ne concerne pas seulement les actants réels, mais aussi les agents virtuels chez qui on travaille à l'émergence d'une conscience artificielle (voir Varela, 1998: 109-112). Dans le cas des systèmes dits intentionnels 5 , les interfaces permettent en effet aux agents artificiels intelligents de se prolonger dans l'espace réel et d'y interpréter, au moyen de filtres perceptuels et psychologiques, les actions et les comportements des organismes vivants.

Les interfaces sont donc des échangeurs sémiotiques pour les deux faces d'un même monde énacté ${ }^{6}$. Elles engendrent ce corps océanique, "continu comme un océan", rêvé par Leibniz et qui déploie les instances dramatiques et narratives sur des scènes virtuelles multidimensionnelles.

\section{INTERACTANTIALITÉ}

On le remarque, le complexe interactif implique une multiplicité d'agents dans l'ontogenèse du signe, et davantage encore dans celle de la narration. Dans cette idée, je proposerai la substitution du terme interactantialité au terme plus général d'interactivité, afin de désigner les situations où, comme l'a souligné Marie-Laure Ryan (2001), le sujet est non pas uniquement en position d'exploration d'un univers prédonné, mais en situation de codétermination d'un monde virtuel?

À cette interactivité numérique de type «ontologique» (Ryan), la notion d'interactantialité adhérera avec force pour quatre raisons essentielles.

En premier lieu, il convient de rappeler la définition que Lucien Tesnière a donné du terme «actant»: «les actants sont les êtres ou les choses qui, à un titre quelconque et de quelque façon que ce soit, même au titre de simples figurants et de la façon la plus passive, participent au procès» (1988: 674). L'indifférenciation fondamentale des actants (humain, animal, objet, média, concept, etc.) confirme la pertinence de ce terme dans un cadre numérique. De plus, dans le procès communicationnel ou narratif, les «actants» confondent habilement le destinateur et le destinataire, le narrateur et le narrataire, les sujets pragmatiques et les sujets cognitifs, les subjectivités humaines et les subjectivités virtuelles intelligentes. On relèvera à ce titre que l'interactantialité numérique fonde l'émergence des mondes virtuels partagés sur la base même de telles fusions ontologiques. Entité multipolarisée par les interfaces, l'actant ne se définit que dans une sphère relationnelle.

En deuxième lieu, la notion d'actantialité confirme l'engagement du corps et de tout l'appareil sensorimoteur dans l'action, orientant cette dernière vers la notion d'énaction définie par Francisco Varela. Nous devons en effet à Varela la description d'une cognition incarnée (embodied cognition), qui, loin de se fonder sur la représentation spéculaire d'un monde prédéfini, se constituerait dans l'interprétation, inscrite à même la corporéité de l'expérience, et d'où émerge la signification créatrice d'un monde. "Énaction", nous dit Varela, décrit «l'avènement conjoint d'un monde et d'un esprit à partir de l'histoire des diverses actions qu'accomplit un être dans le monde» (Varela, Thompson et Rosch, 1993: 35). Ainsi, dans la lignée des travaux de MerleauPonty,

[...] l'idée fondamentale est que les facultés cognitives sont inextricablement liées à l'historique de ce qui est vécu, de la même manière qu'un sentier au préalable inexistant apparaît en marchant. (Ibid.: 111)

Il faudra revenir sur cette approche de la cognition qui apparaitra liée à la structure pré-narrative de l'expérience proposée par Ricœur.

En troisième lieu, l'actantialité se différencie de l'actorialité qui place l'action dans l'ordre de la feintise, du rôle prédéfini et de la posture imitative (voir Schaeffer, 1999). À la différence de l'interacteur, l'interactant est détenteur de fonctions performatives qui, à la fois, décrivent l'ontogenèse du monde virtuel et y participent. Énactant un monde qui ne lui préexiste pas, il est un agent causal au sein d'une chaine d'événements, posant ainsi la prévalence de l'incarnation vécue et générative sur une posture mimétique non causale. 
Examinant l'ontologie du joueur vidéo, Schaeffer pose clairement cette nuance en constatant que, à la différence à la posture actoriale,

[...] la posture d'immersion n'est pas vraiment celle d'une identification allosubjective: le sujet fictionnel qui se déplace

dans l'univers virtuel est en fait un double du joueur réel [...] la

réalité virtuelle dans laquelle je me meus dépend en partie de

mes propres actions. (1999: 314)

Enfin, en quatrième lieu, par le schéma narratif et communicationnel auquel elle renvoie, la notion d'interactantialité renforce l'intimité des liens entre interactivité et mise en intrigue.

\section{MÉSONARRATION}

L'examen d'environnements numériques différents permet de dégager plusieurs degrés de narrativité, depuis la mise en intrigue collaborative jusqu'à l'énaction de mondes virtuels persistants, autopoiétiques et peuplés d'agents intelligents. Pour désigner le trait commun de ces environnements narratifs, on risquera le terme de mésonarrativité, qui renverrait à une narrativité écologiquement située, surgissant du milieu des événements et se déployant elle-même comme milieu.

Parmi les systèmes qui autorisent une mise en intrigue collaborative, deux œuvres retiennent tout d'abord notre attention: In Time de Cyrille C. de Laleu (2005), et Si Poteris Narrare, Licet (2002) de JeanMichel Bruyère.

In Time (2005) est une installation vidéo interactive inspirée de la fiction de Bioy Casares, L'Invention de Morel (1940). Anticipant les dispositifs de Réalité Virtuelle, Bioy Casares imagine un écosystème artificiel grâce auquel des vivants devenus virtuels évoluent sur une île désolée. Un naufragé va à la rencontre de ces simulacres qui répètent, à l'infini, les mêmes dialogues et découvre les machines de projection qui les animent. Amoureux d'un des personnages, il décide d'incorporer le monde virtuel en y transférant sa propre image.

La machine utopique dont rêve Bioy Casares, celle qui, «orientée vers les pensées et les sensations de l'émetteur», permettra de les enregistrer «à n'importe quelle distance» (1973: 97), voire de pénétrer la conscience de l'émetteur, évoque par anticipation les nouvelles machines narratives du virtuel (ibid.). L'art des interfaces, où actants et agents artificiels font émerger ensemble une mésonarration, est l'écho de la machine de fiction de Bioy Casares. La trame invisible du roman - le dispositif de narration collaborative caractérisé par l'interconnexion des machines, des avatars, des vivants et des marées - est cristallisée dans la mise en intrigue interactée d'In Time.

«Collage temporel» 8 de 24 écrans formant le paysage panoramique d'une terre entourée d'eau, In Time redistribue aux visiteurs la multitemporalité de l'île de Bioy Casares, partagée entre l'éternité des personnages virtuels et l'impondérable existence des vivants. Grâce aux discrets capteurs de présence, de position et de déplacement, les visiteurs deviennent les actants de l'univers fictionnel dont, par intrusion de leur propre subjectivité, ils bouleversent la temporalité.

Dans ce dispositif, «la narration devient un événement complexe qui entrelace un nombre d'intersections temporelles et de navigations physiques " ${ }^{9}$. Distribuées par le comportement des sujets dans l'espace de l'installation, les séquences des 24 écrans déforment le paysage vidéographié d'In Time et déploient le drame intime de la réception. Chaque interactant projette, dans les modulations qu'il imprime aux écrans, l'historique de ses propres actions et enchevêtre intimement son histoire virtuelle à celle que sous-tendent les images filmées. Selon le choix et la vitesse de ses déplacements dans l'espace physique, l'unité temporelle des images se disloque d'un écran à l'autre, entraînant la persistance, le ralenti, la disparition des séquences, autrement dit l'agencement des événements dans le lieu même de leur réception.

Avec Michel Serres, on constatera que l'univers fictionnel de Bioy Casares «ne nous est plus livré selon une référence élue par telle ou telle ruse, mais selon un jeu pluraliste d'interférences» (1972: 141). L'historique des actions incarnées est le lieu même d'une mise en 
intrigue énactée. Dans ce cadre, la réception, dont Kendall Walton et Paul Ricœur ont rappelé qu'elle était partie constitutive de la mise en intrigue, devient un modèle dynamique au sein d'une narrativité qui redéploie et partage l'action dans une infinité d'applications, sur une infinité de scènes ouvertes.

Exploitant un système très différent de celui utilisé par Cyrille C. de Laleu, Si Poteris Narrare, Licet (2002) est un environnement vidéo interactif réalisé par JeanMichel Bruyère dans le cadre du projet «EVE" (Extended Virtual Environment, dirigé par Jeffrey Shaw) ${ }^{10}$.

Sphérique, l'espace de projection est un dôme de 12 mètres de diamètre qui permet une projection interne à 360 . Si 124 films composent l'immense fresque virtuelle de Jean-Michel Bruyère, la réception du récit mythique (Diane métamorphosant Actéon) joue sur une mésonarrativité : elle décrit l'histoire conjointe de l'environnement et de ses agents; histoire située qui ne peut surgir que du milieu de la sphère relationnelle des interfaces.

Nunc tibi me posito visam velamine narres, Si poteris narrare, licet. [Va maintenant raconter que tu m'as vue sans voiles, si tu peux le dire, j’y consens.] (Ovide, LIII, 192-195)

Ces paroles de Diane-Artémis, lancées au chasseur Actéon tandis qu'elle le transforme en cerf, s'adressent aussi à la foule des voyeurs du film, à laquelle revient la charge de la mise en intrigue.

Durant six minutes, un seul spectateur est doté d'un casque (tracker frontal) qui projette sur les parois du dôme une fenêtre cinématique où se révèlent les scènes mythologiques. L'interactivité de la projection repose sur un système de tracking qui analyse les mouvements de tête de l'interactant équipé. Si la fresque filmique est virtuellement présente sur l'intégralité de la surface du dôme, seule la zone vers laquelle se focalise l'interactant se dévoile visuellement et auditivement.

Évoquant la puissance d'éclairement de l'Artémis chasseresse (Artemis phæsphôros, «la porteuse de torches» [Callimaque, Hymne à Artémis, 204]), le casque frontal fonctionne alors comme une torche symbolique qui reconfigure le récit. La fenêtre projective qui dévoile les épisodes narratifs est une fenêtre indiscrète, ouverte sur la conscience attentionnelle de l'interactant.

L'intelligence de Si Poteris Narrare, Licet est de transposer l'acte de voyeurisme d'Actéon aux spectateurs qui pénètrent la conscience de l'unique interactant et enchevêtrent à la sienne leur demande de récit. $\mathrm{Si}$, virtuellement, le mythe est disponible dans son ensemble à travers les 124 films juxtaposés, il est sans trajectoire établie et appelle autant de mises en lumière pour autant de recréations. La structure pré-narrative de l'expérience identifiée par Ricour dessine sur les parois du dôme un sentier au préalable inexistant et qui apparaît au fil d'une marche vers le mythe d'un récit. Si poteris narrare, licet rejette en effet toute linéarité ou causalité interne pour mettre en relief une narration énactée, qui s'agence à mi-chemin entre l'histoire potentielle de l'interactant (laquelle se raconte par l'intentionnalité de ses actes) et l'histoire virtuelle d'Artémis multiscénarisée sous le dôme d'«EVE».

D'un tel dispositif qui privilégie l'interlocution des écrans (réel et imaginal) par rapport à la projection unilatérale, l'intersection par rapport à la ligne, Michel Serres dirait qu'il affirme un ars interveniendi. Outre l'absence de continuité temporelle ou causale, c'est cette interréférentialité permanente entre les signes projetés, leur réception et l'interprétation issue de leurs agencements actés, qui singularise la forme narrative proposée par Si Poteris Narrare, Licet.

«Dans le lieu des interréférences, je manque d'une référence globale: il est essentiel que j'en sois privé», écrit Serres (1972: 141). En effet, il s'agit bien d'être privé d'une référence globale, puisque l'ars interveniendi des interfaces se tient dans une nécessaire logique d'interférence qui spectacularise l'action comme productrice d'agencements signifiants.

\section{IDENTITÉ SITUÉE}

Nous ne nous contentons guère d'observer les personnages des cuures de fiction et leurs mouvements. Par le biais du moi fictif, 
nous leur emboittons le pas, nous faisons nôtres leurs soucis, nous nous enfonçons dans le labyrinthe de leur destin.

(Pavel, 1988:184)

À l'évidence, l'intimité ontologique de la personne et du personnage se renforce dans l'interactantialité numérique. Quand, à travers sa présence réelle, le spectateur devient, davantage qu'un élément causal, la source même de production sémiotique de l'univers fictionnel ${ }^{11}$, les statuts ontologiques de l'ensemble des agents narratifs se trouvent puissamment mobilisés.

En une logique métaleptique, les plus subtils glissements de la personne réelle vers le personnage de fiction s'exercent dans les œuvres du Cinéma interactif. Dans ce domaine, Jeffrey Shaw se distingue par la conception de systèmes interactantiels propres à faire émerger de nouvelles "grandes machines» esthétiques.

Le projet "AVIE» (Advanced Visualisation and Interaction Environnement, 2004) ${ }^{12}$, issu des recherches et développements du iCinema, Centre for Interactive Cinema Research (Sydney), déplace les cadres et les frontières conventionnelles de la fiction. Dans cet environnement de visualisation panoramique, stéréoscopique et interactif, les attitudes corporelles et les comportements des spectateurs influent sur les événements de la fiction projetée.

Relié à un logiciel de modélisation 3D, un système de détection infrarouge synthétise en temps réel la présence des sujets dans le cirque de visualisation et les incorpore au monde fictionnel. Les interfaces distribuées permettent alors l'expérience incarnée de la fiction projetée (puisque les événements sonores et visuels changent selon le comportement du sujet dans l'espace interfacé), mais elles autorisent aussi des performances interactées entre des personnes réelles, leurs avatars dans le monde virtuel et les autres personnages fictionnels.

Eavesdrop (2004), une fiction interactive conçue par David Pledger et Jeffrey Shaw pour le système AVIE, joue de ces glissements ontologiques. Dix personnages font le récit de leur vie dans une boucle temporelle que seule pourra modifier l'intrusion du spectateur dans l'espace mental de l'un d'eux. Enchevêtrées les unes aux autres, et dans l'attente d'être racontées, les histoires potentielles des personnages sont révélées dès que le spectateur approche un protagoniste de trop près.

Dans Eavesdrop, la proximité physique rencontre la proximité ontologique; en provoquant la confidence du personnage, le spectateur devient un élément à part entière du monde fictionnel et se confond ontologiquement avec ses interlocuteurs. Dans une telle situation narrative, on constatera, de nouveau avec Ricœur, que les spectateurs

[...] sont donc surélevés au-dessus de leur rôle empirique et

deviennent les figures constitutives d'une intrigue; ils sont métaphorisés, configurés en même temps que l'histoire racontée: il y a configuration des personnages à la mesure de la configuration de l'histoire à laquelle ils contribuent. (2004)

Dans le système AVIE, capture du mouvement et interaction visuelle intelligente ont ainsi pour effet d'engager une «fusion d'horizons» qui pourrait bien contrarier les réserves émises par Pavel vis-à-vis de la possible indistinction du monde réel et de l'univers de la fiction. En effet, face à ces nouvelles machines de fiction, il apparaît difficile d'affirmer encore que «l'espace entre le spectateur et l'œuvre ne sera jamais aboli; les statues hyperréalistes assises sur les bancs des parcs modernes restant toujours des statues" (Pavel, 1988: 185).

Énactées, peuplées d'agents intelligents ou d'avatars guidés par des sujets réels, les fictions numériques ôtent toute pertinence au principe d'un espace fictionnel distinct et distant de l'espace réel. Pour nuancer, nous dirons que, s'il cède d'un point de vue spatiotemporel, le principe de distance, que Pavel tient pour caractéristique de la fiction (ibid.: 183), se déplace symboliquement dans l'étrangeté de l'interactantialité cyberorganique où la fiction numérique fait émigrer le sujet. Le monde de la fiction interactée compense ainsi l'effondrement des frontières avec le réel par l'altérité du monde intersensoriel où il dépayse encore le sujet.

À ce titre, on constatera que l'interaction des personnages fictionnels avec l'actant réel conduit ce 
dernier à l'adoption d'une identité située qui le rapproche des caractéristiques ontologiques du personnage de fiction.

Le film eXistenZ de David Cronenberg (1999) ${ }^{13}$ met en abyme ce procès de fictionnalisation du sujet réel qu'engagent les environnements virtuels. Également jeu virtuel immersif, eXistenZ plonge les protagonistes dans une réalité artificielle où chaque joueur conserve son apparence physique mais intègre la personnalité d'un alter ego fictionnel. S'ils habitent toujours le même corps, les joueurs subissent cependant les effets d'un tropisme artificiel qui les oblige à prononcer des phrases programmées ou à adopter des comportements relatifs à l'identité du personnage fictionnel qu'ils jouent, et qu'ils finissent par devenir.

Ainsi, l'identité des actants paraît située à l'intérieur de la Réalité Virtuelle comme le serait celle d'un personnage de fiction à l'intérieur d'un roman. Comme le confirme Jean-Marie Schaeffer, dans le cas du jeu de Réalité Virtuelle, «le vecteur d'immersion n'est pas celui d'une substitution d'identité physique, mais celui d'une virtualisation de l'identité du joueur» (1999: 314).

\section{INTELLIGENCE}

En faisant des personnages virtuels et des spectateurs les codépositaires de l'acte de narration, les fictions numériques transforment les procès de faire-semblance liés à l'immersion fictionnelle (voir Pavel, 1988: 109-114). Dans l'univers de la fiction interactée, le voyage n'est plus seulement imaginaire, puisque nous faisons bien davantage que "prêter nos corps et nos émotions» (ibid.: 109) ${ }^{14}$ aux personnages fictionnels.

Nos pérégrinations ne sont désormais plus seulement symboliques ${ }^{15}$, et les nouvelles machines de fiction numériques contrarient avec force l'exemple pris par Kendall Walton pour distinguer l'essentielle frontière entre réalité et fiction:

Walton observe que, si nous ne sautons jamais sur scène pour sauver la jeune fille des griffes de son séducteur, c'est que le seul résultat d'un tel acte serait d'interrompre la représentation de la pièce. (Ibid.: 110)
Dans le complexe mésonarratif que nous avons décrit jusqu'à présent, non seulement sommes-nous appelés à intervenir, mais le déficit même de notre intervention mettrait en échec le déroulement de l'intrigue. Nous allons voir que l'intimité de nos rapports avec l'univers fictionnel nous y implique avec d'autant plus de force qu'elle expatrie les entités fictionnelles dans notre propre monde physique et incarné.

L'intégration de l'intelligence artificielle dans les mondes virtuels est sans aucun doute le phénomène qui ouvre le plus de perspectives à la narration et aux procédures de la fiction. La mésonarrativité numérique trouve ainsi des développements conséquents dans les domaines du Jeu vidéo et du Cinéma numérique où l'IA est massivement intégrée.

Avec le développement des MMORPG (Massive Multiplayer Online Role-Playing Game), s'élaborent des «mondes persistants» où l'histoire se déploie en temps réel, à l'échelle d'une interconnexion généralisée et d'une constante régulation de type biologique. Les protagonistes rencontrés à l'intérieur de ces univers fictionnels sont tantôt des joueurs réels représentés par leurs avatars, tantôt des personnages «guidés» par le programme, tantôt, enfin, des agents artificiels autonomes qui enrichissent ensemble les éléments constitutifs d'une fiction habitée.

Dans ces univers perpétuels, personnages joueurs et non joueurs collaborent à l'émergence d'un monde commun avec lequel ils entrent en régulation. Leurs attitudes subjectives viennent nourrir la matrice et permettent ainsi d'éviter un certain déterminisme dans l'historique des événements. Les agents artificiels situés dans le monde virtuel sont en effet dotés de senseurs qui leur permettent de se repérer, d'analyser leur environnement, d'identifier les autres entités et d'interagir avec elles, de prendre en compte la dimension sémantique des actions, d'y associer des décisions imprévisibles en adéquation avec un profil psychologique intégré ou développé au cours de l'histoire.

Dans ces programmes - qui mêlent IA et VA (Vie Artificielle) -, on constatera que le socle narratif joue 
un rôle essentiel dans l'accroissement de la richesse des interactions et le développement des entités intelligentes artificielles. La complexité, qui désigne ici, pour schématiser, les interactions autopoiétiques entre le monde virtuel et le monde réel, est largement favorisée par le contexte narratif qui accroit la signifiance des actions et l'émergence des comportements. La fiction agirait comme opérateur cognitif dans les MMORPG tant pour les actants humains (qui intègrent d'autant mieux le système qu'une histoire rend l'interaction signifiante et gratifiante), que pour les agents artificiels intelligents (qui trouvent dans les mondes fictionnels une richesse environnementale accrue).

Le Cinéma numérique exploite avec bonheur les propriétés de l'IA et son potentiel créateur. Le logiciel de simulation multi-agents Massive (Multiple Agent Simulation System In Virtual Environnement), créé par Stephen Regelous, a ainsi autorisé la réalisation virtuelle d'une bataille de plusieurs milliers d'agents perceptifs et interactifs intelligents ${ }^{16}$. Percevant son environnement et ses congénères, doté de caractéristiques psychologiques propres, chaque agent du système est autonome et produit des variations imprévisibles au sein de l'intrigue. La direction de la scène et une partie de la narration se voient ainsi confiées à des entités abstraites qui y inscrivent une subjectivité inorganique, mais agissante.

Dans ce registre, les systèmes intentionnels développés par les chercheurs du Massachusetts Institute of Technology (MIT) complexifient encore les collaborations humaines et artificielles. Dans la taxinomie des systèmes interactifs, les systèmes dits intentionnels impliquent l'ajout d'un filtre perceptuel (perceptual layer) permettant à l'agent artificiel de se construire un modèle de l'utilisateur humain à partir des données sensorielles que ce dernier lui transmet (gestes, voix, mouvements, positions, expressions faciales, mots, etc.). Au moyen du filtre perceptuel, les données sensorielles sont analysées comme des percepts grâce auxquels l'agent interprète les intentions des interactants réels et s'approche d'un mode humain de communication des émotions.
«[L]'interprétation et les mécanismes du comportement sont influencés par la personnalité de l'agent", selon Sparacino, Davenport et Pentland (1999) ${ }^{17}$, qui va analyser, à l'aune de ses propres caractéristiques émotionnelles, l'intérêt de l'échange avec les sujets humains et adapter ainsi ses réactions face à l'environnement réel.

Prenant en compte la corporéité et la psychologie des actants humains, les agents artificiels sortent donc de leur environnement virtuel pour s'impliquer au sein du monde incarné.

\section{VARIANCE}

Ces nouvelles architectures de l'intelligence changent les procédures narratives en les restructurant à partir de modèles biologiques. Répondant au désir métaleptique qui vise à pénétrer le monde de l'œuvre en devenant l'un de ses personnages, et inversement à faire vivre les entités fictionnelles au sein du monde réel, les mésonarrations numériques sont traversées par les notions d'émergence, d'autonomie et de persistance (laquelle mêle implicitement la notion d'évolution à celle de survie).

Personnages, objets et espaces virtuels acquièrent une précieuse autonomie au moyen des programmes basés sur le comportement (behavior-based), lesquels sont issus des recherches en robotique visant à développer une capacité de réaction rapide face à un environnement dynamique et imprévisible.

C'est cette cognition artificielle énactive qu'évoque Varela lorsqu'il affirme:

[...] si on construit un système qui exprime une grande capacité à

s'incarner, à se développer, dans un cycle d'actions-réactions face

au monde, alors là s'instaure une histoire, qui fait émerger de la signification dans ce monde.

Je n'ai aucun problème [ajoute-t-il] avec l'idée qu'on crée des organismes dotés d'une identité somatique, d'une identité de type sensori-motrice ou d'une conscience artificielle. (1998)

Pour nous, il s'agit de savoir ce que ces agents autonomes, souvent désignés par le terme d'animats (animal + automats), modifient ou créent sur le plan des procédures narratives. Sparacino, Davenport et 
Pentland soulignent tout d'abord le caractère éminemment "ouvert» de la trame narrative proposée par ses concepteurs:

[...] l'avantage des techniques de modélisation basées sur le comportement est que le designer de l'expérience n'a pas à penser à toutes les séquences et les orientations possibles des interactions entre le public et les créatures virtuelles.

(2000: 481) 18

Ainsi, l'introduction de l'IA dans la narrativité numérique affirme ce que j'appellerai un principe de variance, qui désignerait une somme de diversifications impromptues, induites par le comportement autonome des agents en interaction, et qui confère un caractère imprévisible à l'action sans rompre pour autant l'équilibre écosystémique du monde virtuel.

Ce principe de variance induit par les comportements des animats bouscule le principe de distance que Pavel tient pour caractéristique de la fiction (1988). Partageant les mêmes états (autonomie, comportement, personnalité, décision, conscience, etc.) et énactant le même monde dans une temporalité commune, les personnes réelles et les personnages voient leurs différences de statut s'estomper et achèvent de récuser la distance entre monde réel et monde fictionnel ${ }^{19}$. Dans les mondes fictionnels persistants, la distance, encore maintenue, nous l'avons dit, par l'étrangeté inédite des communications cyberorganiques, cédera à terme devant une variance subtilement calquée sur le vivant.

\section{FICTIONS RÉELLES}

Si l'état actuel des recherches nous permet d'envisager la fiction numérique surtout en termes prospectifs, le Cinéma a anticipé l'état avancé de ces mésonarrations intelligentes. En effet, les fictions cinématographiques mettent en scène des sujets humains vivant dans des environnements artificiels, déployés à l'échelle du monde réel.

Dans le film Abre los ojos d'Alejandro Amenábar (1997), le cerveau de César, riche héritier défiguré dans un accident de voiture et dont le corps cryogénisé est relié à un moteur de Réalité Virtuelle, génère un monde fictionnel où il croit vivre réellement. La fiction, comprenant événements, décors, personnages et motifs, émerge de l'esprit de César couplé aux interfaces comme la projection vivante de son "histoire potentielle», version idéalisée d'une seconde vie.

Tandis que la Réalité Virtuelle incorpore habituellement le sujet dans un monde virtuel qui lui préexiste partiellement, la réalité inversée dans laquelle vit César fait du sujet l'unique matrice de son univers sémiotique. Cependant, les événements de cette "fiction réelle» ne sont pas agencés par le seul esprit de César: la présence de personnages herméneutiques qui interviennent aux moments clés de l'action révèle la collaboration d'agents artificiels dans la mise en intrigue.

La réalité inversée de César, si éloignée soit-elle d'une situation techniquement réalisable aujourd'hui, évoque une inversion polaire qui n'est pas sans conséquence sur les procédures narratives et fictionnelles. Principal personnage d'une fiction réelle, César est guidé par des agents artificiels dont la posture herméneutique au sein du monde virtuel leur confère le rôle "d'actant primitif du système" (Sparacino, Davenport, Pentland, 2000: 483; je traduis), voire de narrateur. Des recherches récentes tendent à confirmer cette mutation.

Nés dans les laboratoires du MIT, les systèmes intentionnels appelés media actors sont des signes virtuels doués d'une forme de "conscience artificielle» dont l'évolutivité promet de renforcer la transformation des écritures et des protocoles narratifs. Images, vidéos, graphes, sons, textes et discours intègrent un programme d'intelligence artificielle dont les propriétés perceptuelles permettent de leur assigner un comportement autonome et une capacité d'interaction complexe avec le monde réel. N'importe quel média peut ainsi se voir attribuer des compétences sensorielles, des traits de personnalité et des états sentimentaux qui influent sur le degré et la nature des interactions avec les sujets humains. 
Les media actors sont modélisés comme des agents logiciels dont la personnalité n'affecte non seulement leur état interne (sentiments), mais aussi leur perception $d u$ comportement $d u$ public (intentions) et leur suppositions quant à leurs interactions futures avec les interacteurs humains. (Ibid.) 20

Ces développements permettent alors des interactions très riches en temps réel avec des ensembles sémiotiques doués de compétences analytiques, affectives et psychologiques qui affecteront tous les niveaux des narrations interactées.

Les arts numériques et les univers virtuels multijoueurs nous ont permis de cerner certaines modalités et fonctions de la narration interactive. Parmi celles-ci, nous avons identifié les propriétés de la fiction numérique qui contribuent à l'émergence et à la persistance des mondes virtuels; enfin, nous avons révélé un principe de variance qui confirme la proposition d'une mésonarrativité.

Dans ce contexte, on présagera de l'émergence de fictions comme entités cognitives, intelligentes et autopoïétiques, qui redéfiniront profondément notre expérience incarnée.

\section{N O TES}

1. «L'organisme n'est pas seulement sa réalité locale-instantanée, pour une pensée proximale, ni d'ailleurs une autre réalité. Il est “phénomène-enveloppe", macroscopique, que l'on n'engendre pas à partir des éléments, qui investit le local-instantané, qui n'est pas à chercher derrière, mais entre les éléments " (Merleau-Ponty, 1994: 275, souligné par l'auteur).

2. Selon l'expression de Derrick de Kerckhove (2000).

3. J'ai analysé l'adhérence de la métaphore de l' "environnement" numérique au modèle biologique du «milieu " (voir Morignat, 2004). 4. Voir le site de Electronic Shadow: http://

www.electronicshadow.com (page consultée le 27 avril 2006); celui de Intro Act: http://www.medienkunstnetz.de/works/intro-act/ (consultée le 3 mai 2006) et http://www.interface.ufg.ac.at/christalaurent/WORKS/index.html (consultée le 9 mai 2006).
5. Que j'aborderai dans la dernière partie de cet article.

6. Selon la théorie de l'énaction de F. Varela évoquée plus loin.

7. "In the exploratory mode, the user is free to move around the database, but this activity does not make history nor does it after the plot; the user has no impact on the destiny of the virtual world. In the ontological mode, by contrast, the decisions of the user send the history of the virtual world on different forking paths. These decisions are ontological in the sense that they determine which possible world, and consequently which story will develop from the situation in which the choice presents itself" (Ryan, 2001).

8. En ligne, http://nujus.net/ cyrille/InTime.htm (page consultée le 27 avril 2006).

9. Brown, Del Favero et Weibel, 2003: 315. C'est moi qui traduis: "Narrative becomes a complex event which interlaces a number of intersecting temporal and physical navigations".

10. Le iCinema a été développé par Jeffrey Shaw au sein du ZKM, Institut des Médias Visuels de Karlsruhe. Voir en ligne: http:// epidemic.cicv.fr/geo/art/jmb/prj/i_cinema/index.html (page consultée le 3 mai 2006).

11. Tel est le cas dans les systèmes de «Réalité Inversée » où les signes visuels et sonores sont entièrement générés par la corporéité interfacée du sujet, ou encore dans les œuvres de Réalité Augmentée comme Pico Scan de Sommerer et Mignonneau, où toutes les informations nécessaires à la génération visuelle sont saisies directement sur le corps des actants.

12. Le projet AVIE (2004) résulte d'une collaboration de Jeffrey Shaw avec Dennis Del Favero. En ligne: http://www.icinema.unsw.edu.au/ (page consultée le 27 avril 2006). AVIE y est décrit: «[...] Panoramic Immersive Stereoscopic 3D Visualization System which consists of a cylindrical silvered screen 4 metres high and 10 metres in diameter on whose internal surface $360^{\circ}$ stereographic $3 \mathrm{D}$ panoramic image sequences can be projected".

13. Pour plus de détails à propos d'eXistenZ, voir en ligne: http:// www.objectif-cinema.com/analyses/050.php (page consultée le 9 mai 2006).

14. Pavel poursuit: "Nous envoyons nos moi fictionnels reconnaître le territoire avec l'ordre de rédiger aussitôt un rapport; ils sont émus, non pas nous, ils ont peur de Godzila et pleurent avec Juliette, alors que nous ne faisons que leur prêter nos corps et émotions, un peu comme dans les rites chamaniques les fidèles prêtent leurs corps aux esprits bienfaisants " (ibid., je souligne).

15. «[N]os pérégrinations sont purement symboliques" (ibid.). 16. Voir la bataille d'Helm dans Le Seigneur des anneaux (film de P. Jackson, 2003).

17. Je traduis: "Both the interpretation and the behavioral mechanisms are influenced by the personality of the agent" (Sparacino, Davenport et Pentland, 1999: 3).

18. Je traduis: "The advantage of behavior-based modeling techniques is that the designer of the experience does not have to think of all the possible sequences and branchings in defining the interaction between the public and the virtual creatures".

19. Voir à ce sujet les recherches sur les acteurs perceptifs interactifs et les humains virtuels distribués du MIRALab (laboratoire d'infographie de l'Université de Genève, dirigé par N. Magnenat Thalmann). 20. Je traduis : "Media actors are modeled as software agents whose personality affects not only their internal state (feelings) but also their perception of the public's behavior (intentions) and their expectations about future interactions with their human interactor". 


\section{RÉFÉREN CES BIBLIO G RAPH IQ U ES}

Bioy CASARES, A. [(1940) 1973]: L'Invention de Morel, Paris, Gallimard, coll. " $10 / 18$ ".

Brown, D., D. Del Favero, J. ShaW, P. Weibel [2003] : "Interactive Narrative as a Multi-Temporal Agency", dans J. Shaw et P. Weibel (dir.), Future Cinema: The Cinematic Imaginary after Film, Cambridge (Mass.), The MIT Press, 312-315.

DiCK, P. K. [1988]: Le Grand O, Paris, Denoël.

KeRCKHOVE, D. de [(1997) 2000]: L'intelligence des réseaux, Paris, Odile Jacob.

LAVAL, C. [1994]: Jeremy Bentham. Le Pouvoir des fictions, Paris, PUF, coll. "Philosophies".

MERleau-PonTY, M. [1995]: La Nature. Notes de cours du Collège de

France (textes établis et annotés par D.Séglard), Paris, Seuil.

MORIGNAT, V. [2004]: «Environnements virtuels et nouvelles stratégies actantielles", Arts de la scène, scène des arts, vol. III (Formes hybrides : vers de nouvelles identités), textes réunis par L. Boucris et M. Freydefont,

Études théâtrales, no 30, École d'Architecture de Nantes - Centre

d'études théâtrales de Louvain. En ligne: http://

www.valeriemorignat.net.

PAVEL, T., [1988]: Univers de la fiction, Paris, Seuil.

RICCEUR, P., [1983]: L'Intrigue et le récit historique, Temps et Récit (tome 1), Paris, Seuil;

[2004]: Arts, langage et herméneutique esthétique (entretien avec

J.-M. Brohm et M. Uhl). En ligne: http://www.philagora.net/philo- fac/ricoeur.htm (page consultée le 27 avril 2006).

RYAN, M.-L. [2001]: "Beyond myth and metaphor: The case of narrative in digital media ", Games Studies, International Journal of Computer Game Research, vol. I, no 1 (juillet). En ligne: http:// www.gamestudies.org/0101/ryan (page consultée le 6 juin 2006). SchaefFer, J.-M. [1999]: Pourquoi la fiction?, Paris, Seuil. SERres, M. [1972] : Hermès II. L'interférence, Paris, Minuit. Sparacino, F., G. DaVEnPorT et A. PEnTland [1999]: "Media Actors: Characters in Search of an Author ", IEEE Multimedia Systems '99, International Conference on Multimedia Computing and Systems, Centro Affari, Florence, 7-11 juin. En ligne : http://

web.media.mit.edu/ flavia/Papers/mediaActors.pdf (page consultée le 3 mai 2006);

[2000]: «Media in performance: Interactive spaces for dance, theater, circus, and museum exhibits ", IBM Systems Journal, vol. 39, $n^{\text {os } 3-4, ~ 479-510 . ~ E n ~ l i g n e ~: ~ h t t p: / / w w w . r e s e a r c h . i b m . c o m / j o u r n a l / s j / ~}$ 393/part1/sparacino.html (page consultée le 3 mai 2006).

TESNIÈRE, L. [(1959) 1988]: Éléments de syntaxe structurale, Paris, Klincksieck.

VARELA, F. [1998]: «Entretien avec Francisco Varela ", propos recueillis par H. Kempf, La Recherche, no 308, avril, 109-112. En ligne: http:// www.overdream.com/html/varela.htm (page consultée le 27 avril 2006).

VARELA, F., E. THOMPSON et E. ROSCH [1993]: L'Inscription corporelle de l'esprit. Sciences cognitives et expérience humaine, Paris, Seuil. 